\title{
Las empresas de inserción en la economía social y solidaria: perspectivas y propuestas desde FAEDEI
}

\author{
Insertion companies in social and solidarity economy: \\ perspectives and proposal from FAEDEI \\ María Elena Gil BARTOLOMÉ \\ Asociación Madrileña de Empresas de Inserción \\ elena.gil@fundacionsmp.org \\ Nieves RAmos Rosario \\ Federación de Asociaciones Empresariales de Empresas de Inserción \\ secretaria@faedei.org
}

Recibido: 22/11/2012

Revisado: 04/12/2012

Aceptado: 09/01/2013

Disponible on line: 16/04/2013

\section{Resumen}

El objetivo del artículo es presentar las perspectivas y propuestas que se ponen en marcha en la Federación de Asociaciones Empresariales de Empresas de Inserción (FAEDEI), para fomentar la visibilización, el desarrollo y la creación de empresas de inserción. El artículo explica el marco de actuación en el que las más de doscientas empresas de inserción que actualmente operan en España desarrollan su modelo de economía social, de eficacia reconocida para la inserción sociolaboral de personas con dificultades de acceso al mercado laboral.

Palabras clave: empresas de inserción, economía social, inserción sociolaboral

\begin{abstract}
Main goal of the paper is to introduce new perspectives and proposals put into commission by the Insertion Companies Entrepreneurial Associations Federation (Federación de Asociaciones Empresariales de Empresas de Inserción,FAEDEI) to promote the visibility, development and creation of insertion companies. The paper explains the action frame in which the more than two hundred insertion companies currently operating in Spain develop their social economy model, o model of recognized efficiency for the social and labour insertion of persons with difficulties to access the labour market. Keywords: insertion companies, social economy, social and labour insertion.
\end{abstract}

Referencia normalizada: Gil Bartolomé, M. E., y Ramos Rosario, N. (2013): «Las empresas de inserción en la economía social y solidaria: perspectivas y propuestas desde FAEDEI». Cuadernos de Trabajo Social, 26(1); 43-53.

Sumario: Introducción. 1. La ley 5/2011, de Economía Social. 2. La ley 5/2011, de Economía social y las empresas de inserción. 3. La Estrategia de empleo 2012-2014 y las empresas de inserción. 4. Responsabilidad social empresarial y empresas de inserción. 5. El proceso de acompañamiento en las empresas de inserción. 6. Conclusiones. 7. Referencias bibliográficas.

\section{Introducción}

La Federación de Asociaciones Empresariales de Empresas de Inserción (FAEDEI), es una entidad sin ánimo de lucro de ámbito estatal, que nace a finales del año 2007 como la única representante de las empresas de inserción en España.

Las empresas de inserción son un modelo de economía social de eficacia reconocida para la inserción sociolaboral de personas con dificultades de acceso al mercado laboral. Son un instrumento importante para la puesta en práctica de las políticas activas de empleo ya que de manera autónoma y económicamente viable ofrecen itinerarios personalizados de formación, empleo e inserción sociolaboral a personas en riesgo o situación de exclusión social.

FAEDEI agrupa a las asociaciones empresariales de empresas de inserción existentes en 
todo el territorio nacional. Actualmente está compuesta por las siguientes asociaciones:

- Asociación de Empresas de Inserción de Canarias, ADEICAN.

- Asociación de Empresas de Inserción del Principado de Asturias, ADEIPA.

- Asociación de Empresas de Inserción de Galicia, AEIGA.

- Asociación de Empresas de Inserción de Madrid, AMEI.

- Asociación Aragonesa de Empresas de Inserción, AREI.

- Asociación de Empresas de Inserción de Castilla-La Mancha, ASEIRCAM.

- Asociación Valenciana de Empresas de Inserción, AVEI.

- Asociación de Empresas de Inserción de Andalucía, EIDA.
— Federación de Empresas de Inserción de Cataluña, FEICAT.

- Federación Castellano-Leonesa de Empresas de Inserción, FECLEI.

- Asociación de empresas de Inserción del País Vasco, GIZATEA.

- Asociación de Empresas de Inserción de Extremadura, INSERCIONEX.

La Federación mantiene contactos con las empresas de inserción de las comunidades autónomas de Murcia, Cantabria e Islas Baleares, en proceso de constitución de sus agrupaciones territoriales, así como con la Asociación de Centros de Inserción de Navarra, que están participando en las actividades organizadas por la Federación.

A continuación presentamos en el Tabla 1 los datos más significativos de las empresas de

\begin{tabular}{|c|c|}
\hline Total de empresas de inserción que han participado en la memoria social & 167 \\
\hline \multicolumn{2}{|l|}{ Datos referidos a las personas } \\
\hline Total de personas trabajadoras —inserción y no inserción- & 4.563 \\
\hline Personas trabajadoras de inserción $(*)$ & 2.435 \\
\hline Total puestos equivalentes a jornada completa —inserción y no inserción- & 2.478 \\
\hline Puestos de inserción equivalentes a jornada completa & 1.329 \\
\hline Porcentaje de personas que acceden al mercado laboral una vez finalizado su itinerario & $52 \%$ \\
\hline \multicolumn{2}{|l|}{ Datos económicos } \\
\hline Ingresos totales (euros) & $83.279 .169,46$ \\
\hline Facturación Total (euros) & $62.681 .850,58$ \\
\hline Porcentaje de ayudas públicas sobre ingresos de explotación & $20,11 \%$ \\
\hline Costes en personal & $51.018 .319,95$ \\
\hline Valor añadido bruto generado & $53.148 .993,06$ \\
\hline \multicolumn{2}{|l|}{ Retorno económico de las empresas de inserción a las administraciones públicas } \\
\hline $\begin{array}{l}\text { Estimación del retorno a las administraciones públicas en concepto de Seguridad } \\
\text { Social, IRPF, IVA o situaciones especiales (IGIC), Impuesto sobre Sociedades } \\
\text { y otros tributos }\end{array}$ & $\begin{array}{l}9.209 .052,84 € \\
6.929,52 € \\
\text { por plaza } \\
\text { de inserción }\end{array}$ \\
\hline
\end{tabular}

Tabla 1. Datos significativos de las Empresas de Inserción asociadas a FAEDEI durante 2011.

Nota: * Diferenciamos entre puesto y persona en inserción: un puesto o plaza de inserción implica una jornada completa de trabajo, mientras una persona puede ocupar un puesto a jornada completa o tener una dedicación parcial.

Fuente: FAEDEI. 
inserción que han participado en nuestra Memoria de balance social 2011.

\section{La ley 5/2011, de Economía Social}

La configuración actual de la economía social española viene marcada por la aprobación de la Ley 5/2011, de 29 de Marzo, de Economía Social, que supone un reconocimiento de todas las familias de este modelo de desarrollo económico, aportándoles visibilidad y posibilitando el desarrollo del sector, tanto en el marco del Estado como en la Unión Europea. La ley de Economía social reconoce un conjunto empresarial diverso que se agrupa en las familias (Cuadro 1) que presentan rasgos propios que facilitan el cumplimiento de sus fines diversos y les permiten cumplir de forma adecuada con su compromiso social con sus grupos de interés.

Todas ellas comparten los principios que orientan la economía social en España:

- Primacía de las personas y del fin social sobre el capital, que se concreta en una gestión innovadora, sostenible, autónoma, transparente, democrática y participativa. Las empresas de economía social priorizan la toma de decisiones más en función de las personas y sus aportaciones de trabajo y servicios prestados a la entidad o en función del fin social, que en relación a sus aportaciones al capital social.

- Están legalmente constituidas sin ánimo de lucro, por lo que aplican los resultados obtenidos de la actividad económica principalmente al fin social objeto de la entidad.

- Promocionan la solidaridad interna y con la sociedad, favoreciendo el compromiso con el desarrollo local, la igualdad de oportunidades entre hombres y mujeres, la cohesión social, la inserción de personas en riesgo de exclusión social, la generación de empleo estable y de calidad, la conciliación de la vida personal, familiar y laboral y la sostenibilidad.

- Son independientes respecto a los poderes públicos, se centran en prestar servicios allí dónde la administración pública o el sector lucrativo fallan en su provisión.

Son también señas de identidad comunes de estas familias la capacidad de innovación para satisfacer los problemas que surgen en la sociedad, y la defensa de cambios sociales, legales, administrativos, o de otro tipo, siempre en defensa de los derechos y las libertades de las personas, con base en el respeto a la diversidad, la pluralidad y la tolerancia.

\section{La ley 5/2011, de Economía social y las empresas de inserción}

Las empresas de inserción consiguieron su regulación en la Ley 44/2007 de 13 de Diciembre del 2007, con el apoyo de todos los partidos políticos presentes en el arco parlamentario. La propuesta legislativa y la negociación con los responsables políticos se hizo en el seno de la Confederación Española de la Economía Social (CEPES), teniendo como representantes de la Administración Pública a la Dirección General de Economía Social, Autónomos y Responsabilidad Social Empresarial.

Con esta normativa, se les ratifica a las empresas de inserción como instrumentos de inserción por lo económico de los colectivos vulnerables, dentro de un tipo de economía que tiene como centro a la persona, el bien común, y desarrollando un papel fundamental en estos momentos de crisis, cuando se manifiesta la necesidad de proponer nuevos modelos productivos sostenibles y respetuosos con el medio ambiente, y que tengan en cuenta la necesaria igualdad de oportunidades.

Esta filosofía de empresa social, sostenible y autónoma, que muchos analistas plantean como necesaria ante el nuevo escenario económico, ha estado presente a lo largo del desarrollo de este modelo de empresa, siendo las señas de identidad de las empresas de inserción desde su origen. A lo largo de estos años se han ido creando y mejorando los procesos metodológicos que hacen eficaces la permanente y necesaria relación de la formación integral de las personas en el ámbito laboral con un acompañamiento específico y personalizado. La actualmente conocida y popularizada como «formación dual a la alemana» es el modelo pedagógico definido y desarrollado por las empresas de inserción a lo largo de toda su trayectoria, a través de los itinerarios personalizados ya recogidos en las leyes y decretos correspondientes y confirmados por los registros autonómicos, implantada desde hace años y $\sin$ necesidad de busca fuera lo que ya funciona en España.

Por este motivo, el espaldarazo que aportan las dos leyes que regulan las empresas de in- 


\begin{tabular}{|c|c|}
\hline Familia & Rasgos propios \\
\hline Cooperativas & $\begin{array}{l}\text { Son una forma de organización empresarial basada en la estructura y funcionamiento } \\
\text { democráticos. Su actividad se desarrolla atendiendo a los principios cooperativos, } \\
\text { aceptados y regulados en los ámbitos autonómico, estatal e internacional: la adhesión } \\
\text { voluntaria y abierta de los socios, la gestión democrática, la participación económica } \\
\text { de los socios, la educación, formación e información y el interés por la comunidad. }\end{array}$ \\
\hline $\begin{array}{l}\text { Sociedades } \\
\text { laborales }\end{array}$ & $\begin{array}{l}\text { Han mostrado un alto potencial de generación de empresas. En este tipo de empresas, } \\
\text { el capital social pertenece mayoritariamente a las personas trabajadoras. El hecho de } \\
\text { que sean socias favorece la automotivación a la hora de afrontar los proyectos. El mí- } \\
\text { nimo requerido es de tres, y los trámites de constitución son similares a los de cual- } \\
\text { quier otra sociedad mercantil. }\end{array}$ \\
\hline Mutualidades & $\begin{array}{l}\text { Son sociedades de personas, sin ánimo de lucro, de estructura y gestión democrática, } \\
\text { que ejercen una actividad aseguradora de carácter voluntario, complementaria del sis- } \\
\text { tema de previsión de la Seguridad Social }\end{array}$ \\
\hline $\begin{array}{l}\text { Cofradías de } \\
\text { pescadores }\end{array}$ & $\begin{array}{l}\text { Son corporaciones de derecho público sectoriales, sin ánimo de lucro, representativa } \\
\text { de intereses económicos de armadores de buques de pesca y de trabajadores del sec- } \\
\text { tor extractivo, que actúan como órganos de consulta y colaboración de las administra- } \\
\text { ciones competentes en materia de pesca marítima y de ordenación del sector pesque- } \\
\text { ro, cuya gestión se desarrolla con el fin de satisfacer las necesidades e intereses de sus } \\
\text { socios, con el compromiso de contribuir al desarrollo local, la cohesión social y la } \\
\text { sostenibilidad. }\end{array}$ \\
\hline $\begin{array}{l}\text { Centros } \\
\text { especiales de } \\
\text { empleo }\end{array}$ & $\begin{array}{l}\text { Son empresas que compatibilizan la viabilidad económica y su participación en el } \\
\text { mercado con su compromiso social hacia colectivos con menores oportunidades en el } \\
\text { mercado de trabajo. Su plantilla está constituida por el mayor número de personas con } \\
\text { discapacidad (cuyo número no puede ser inferior al } 70 \% \text { respecto del total de los tra- } \\
\text { bajadores). Desarrollan una capacidad productiva y competitiva que les permite intro- } \\
\text { ducir sus productos en el mercado. }\end{array}$ \\
\hline Asociaciones & $\begin{array}{l}\text { Sobre todo las vinculadas al movimiento de la discapacidad y de la inserción de per- } \\
\text { sonas en exclusión. Las principales características de este movimiento asociativo se } \\
\text { centra en prestar servicios allí dónde el sector lucrativo falla en su provisión, que ade- } \\
\text { más suele coincidir con aquellos sectores en los que se satisfacen derechos fundamen- } \\
\text { tales, sobre todo en su acceso a colectivos especialmente vulnerables, como las per- } \\
\text { sonas con disparidad. }\end{array}$ \\
\hline Fundaciones & $\begin{array}{l}\text { Son organizaciones constituidas sin fin de lucro que, por voluntad de sus creadores, } \\
\text { tienen afectado su patrimonio de modo duradero a la realización de un fin de interés } \\
\text { general. Las fundaciones de Economía Social deben cumplir taxativamente los prin- } \\
\text { cipios de la Economía Social citados, y que recoge la Ley } 5 / 2011 \text {. }\end{array}$ \\
\hline $\begin{array}{l}\text { Empresas de } \\
\text { inserción }\end{array}$ & $\begin{array}{l}\text { Se definen como «estructuras de aprendizaje, en forma mercantil, cuya finalidad es } \\
\text { la de posibilitar el acceso al empleo de colectivos desfavorecidos, mediante el des- } \\
\text { arrollo de una actividad productiva, para lo cual, se diseña un proceso de inserción, } \\
\text { estableciéndose durante el mismo una relación laboral convencional». En su plantilla } \\
\text { deben tener un porcentaje de colectivos en inserción, entre el } 30 \% \text { y el } 50 \% \text {. El } 80 \% \\
\text { de los resultados se reinvierte en la empresa. }\end{array}$ \\
\hline
\end{tabular}

Cuadro 1. Familias de empresas reconocidas en la Ley 5/2011, de Economía social.

Fuente: Elaboración propia.

serción y reconocen su pertenencia a la economía social, debe servir a las empresas de inser- ción para hacer frente a uno de sus retos más importantes: su falta de visibilidad y la necesi- 
dad de poner en valor las buenas prácticas que realizan como empresas de economía social.

Las empresas de inserción durante estos años han consolidado su modelo estructural de doble vertiente, mercantil y social, al tiempo que negociaban con la Administración Pública la implantación de los registros autonómicos adaptados a la ley que proporcionan su calificación. En la mayoría de los casos ha sido un proceso complejo debido a la escasez, cuando no ausencia, de medidas de apoyo y promoción, que han requerido el esfuerzo y el compromiso de personas y entidades promotoras convencidas de la idoneidad de este modelo empresarial.

Es necesario poner de manifiesto «la falta de madrinas y padrinos» reconocidos para muchos de los colectivos en situación o riesgo de exclusión. Frente a la gran concienciación social que presenta el colectivo de las personas con discapacidad (consiguiendo la Ley de Integración Social de los minusválidos (Ley 13/1982, de 7 de abril de Integración social de los minusválidos, LISMI), los centros especiales de empleo, los enclaves laborales, con un importante apoyo institucional y respaldo económico) hay otros colectivos como los jóvenes, mayores de dieciocho años y menores de treinta, procedentes de instituciones de protección de menores, las personas con problemas de drogodependencia $\mathrm{u}$ otros trastornos adictivos que se encuentren en proceso de rehabilitación o reinserción social o las personas internas en centros penitenciarios que no cuentan con el mismo respaldo social. Todos estos colectivos tienen la necesidad de insertarse o reinsertarse laboralmente, sobre todo en una sociedad como la nuestra en la que el empleo se convierte en uno de los principales indicadores de una sociedad saludable, en términos de educación, bienestar e igualdad de oportunidades.

Por ello, se entiende que sean las entidades sociales que trabajan con los colectivos con necesidades de inclusión quienes «realicen ese madrinazgo o padrinazgo» y se conviertan en promotoras de las empresas de inserción, conscientes, por un lado, de la dificultad de las personas de acceder directamente al mercado laboral sin haber realizado un itinerario personalizado de formación, y por la otra del rechazo que estas personas pueden padecer $a$ priori debido a su procedencia.
Por este motivo, es de vital importancia para las empresas de inserción, en este momento de enormes dificultades de acceso al empleo y de un aumento del riesgo de exclusión de las personas más vulnerables, visibilizarlas. Sobre todo teniendo en cuenta que los colectivos trabajadores de las empresas de inserción, recogidos en la Ley 44/2007, de 13 de diciembre, para la regulación del régimen de las empresas de inserción, además de los anteriormente citados, son cada vez más numerosos como: los perceptores de rentas mínimas de inserción, o cualquier otra prestación de igual o similar naturaleza y las personas que no puedan acceder a las prestaciones por falta del período exigido de residencia o empadronamiento o haber agotado el período máximo de percepción de prestaciones legalmente establecido.

\section{La Estrategia de empleo 2012-2014 y las empresas de inserción}

La publicación de la Estrategia de Empleo 2012-2014 el Real Decreto 1542/2011, de 19 de Noviembre de 2011, suponen un hito de referencia para la puesta en marcha de proyectos y programas de las empresas de inserción, ya que se fija como uno de los diez principales ámbitos de actuación: la promoción de la inclusión social, la lucha contra la pobreza y la generación de oportunidades para colectivos con especiales dificultades. Concretamente en el punto 5.6 se hace referencia a la necesidad de articular medidas que contribuyan a impulsar la contratación de personas en situación de exclusión social a través de las empresas de inserción.

Los proyectos empresariales y programas de acción que estamos construyendo desde las empresas de inserción se incluyen dentro del marco normativo que ofrecen tanto el Ministerio de Empleo y Seguridad Social como el de Sanidad, Servicios Sociales e Igualdad.

Por su especial importancia e incidencia sobre la actividad de las empresas de inserción, debemos destacar medidas normativas como la Ley de Cualificaciones y de la Formación Profesional (Ley Orgánica 5/2002, de 19 de junio) y la Ley $3 / 2012$, en la que se regula el contrato de formación y aprendizaje en las empresas de inserción. Ambas normativas ofrecen un marco de actuación, una oportunidad de desarrollo que contribuye a reducir los obstáculos 
de entrada al mercado de trabajo a colectivos en situación o riesgo de exclusión puesto que reconocen un sistema para certificar y cualificar oficialmente de los aprendizajes que se producen en los procesos formativos de las empresas de inserción.

La reciente publicación del Real DecretoLey 4/2013, de 22 de febrero, de medidas de apoyo al emprendedor y de estímulo del crecimiento y de la creación de empleo, que desarrolla la Estrategia de Emprendimiento y Empleo Joven 2013-2016, recoge en su Capítulo III, de los estímulos a la contratación, referencias que incentivan la incorporación de jóvenes a las empresas de inserción. Se trata, en concreto, del artículo 14, el que recoge estas nuevas medidas incentivadoras (Cuadro 2).

Este marco normativo debe utilizarse para visibilizar, el instrumento en sí, de las empresas de inserción ante la sociedad y la Administración Pública que debe facilitar los mecanismos de certificación, registros para el mantenimiento y creación de nuevas empresas y medidas de apoyo que equilibren el sobrecoste económico que supone la inserción sociolaboral de personas en riesgo o situación de exclusión. En este sentido, contamos con indicadores incluidos en nuestra memoria de balance social que miden el retorno económico que generan las empresas de inserción a las administraciones públicas. Para ello, se analizan diferentes magnitudes económicas como son los pagos a la seguridad social, IRPF, IVA, el impuesto de sociedades y otros tributos. En el balance social del año 2011, las empresas de inserción que participaron en el informe generaron un retorno económico de 9.209.052 euros por los 1.329 puestos de inserción con los que cuentan, esto supone un retorno económico para la Administración Pública de 6.929,3 euros por cada puesto de trabajo. Estas cifras demuestran la eficacia y rentabilidad de las empresas de inserción como herramientas de las políticas activas de empleo frente a las políticas pasivas.

\section{Responsabilidad social empresarial y empresas de inserción}

La Comisión Europea presentó en 2011 la Estrategia renovada sobre la responsabilidad social de las empresas en la que se incluye una nueva definición de la responsabilidad social empresarial, a saber: «la responsabilidad de las empresas por su impacto en la sociedad» (COM, 2011, 681 final). El respeto de la legislación aplicable y de los convenios colectivos entre los interlocutores sociales, es un requisito previo al cumplimiento de dicha responsabilidad.

Con el fin de favorecer una «economía social de mercado altamente competitiva», la Comisión Europea situó la economía social y la innovación social en el centro de sus iniciativas, tanto en lo que concierne a la cohesión territorial como a la búsqueda de soluciones originales para los problemas sociales $\mathrm{y}$, en concreto, en la lucha contra la pobreza y la exclusión, en su Estrategia Europa 2020 (COM, 2010), en la iniciativa emblemática titulada «Unión por la innovación» (COM, 2010, 546 final), en la Plataforma Europea contra la Pobreza y la Exclusión Social (COM 2010, 578 final) y en el Acta del Mercado Único (COM, 2011,206 final). El mercado único necesita un nuevo crecimiento inclusivo y orientado hacia el empleo para todas las personas. Se trata de responder así a la demanda creciente de la ciudadanía de que su trabajo, su consumo, su ahorro y sus inversiones tengan una repercusión y un sentido más «éticos» y más «sociales».

\section{Artículo 14. Incentivos a la incorporación de jóvenes a entidades de la economía social}

1. Se incorporan las siguientes bonificaciones aplicables a las entidades de la economía social:

b) Bonificaciones en las cuotas de la Seguridad Social aplicables a las empresas de inserción en los supuestos de contratos de trabajo suscritos con personas menores de 30 años en situación de exclusión social incluidas en el artículo 2 de la Ley 44/2007, de 13 de diciembre, para la regulación del régimen de las empresas de inserción, de 137,50 euros/mes (1.650 euros/año) durante toda la vigencia del contrato o durante tres años, en caso de contratación indefinida. Estas bonificaciones no serán compatibles con las previstas en el artículo 16.3.a) de la Ley 44/2007, de 13 de diciembre.

Cuadro 2. Estímulos a la contratación (art. 14).

Fuente: Elaboración propia. 
Dentro del Plan estratégico 2009-2011 de FAEDEI se incluyó una línea de acción para la creación de programas de promoción y visibilización de los modelos de gestión que desarrollan las empresas de inserción, socialmente responsables y comprometidos con la realidad de sus territorios, siendo su principal objetivo: dar a conocer los productos y servicios de las empresas de inserción a todos los potenciales colaboradores o compradores. Para el desarrollo de esta línea de acción se creó el Proyecto Empresa/Entidad Responsable con la Inserción (ERI) un esquema de certificación privado con el que la Federación reconoce la responsabilidad social con la inserción de empresas y administraciones públicas que colaboran con las empresas de inserción a través de dos acciones concretas:

1. La contratación de productos y servicios con las empresas de inserción, que nos permitiría mantener los puestos de trabajo actuales, así como crear nuevas empresas y nuevos puestos de trabajo.

2. Realizar conjuntamente el tránsito de quienes han completado ya con éxito su proceso en las empresas de inserción, ofreciéndoles a las empresas a personas formadas, motivadas y fundamentalmente agradecidas por continuar activas en el mercado laboral, y por seguir con su derecho de ciudadanía plena que da el trabajo.

El proyecto ERI cuenta con el apoyo del Ministerio de Empleo, el de Sanidad, Servicios Sociales e Igualdad y de la Fundación «La Caixa». Durante la celebración de INSERCIONA, la II Feria de Empresas de Inserción en Irún, del 31 de Enero al 1 de Febrero de 2013, se realizó la primera entrega de premios y certificados ERI a aquellas empresas, entidades sin ánimo de lucro y administraciones públicas que han contribuido a la puesta en marcha del certificado. Actualmente el Proyecto ERI ha adherido a más de 50 empresas y entidades colaboradoras, entre las que se encuentran: el Instituto Aragonés de Empleo (INAEM), el Gobierno Vasco, la Consejería de Economía, Hacienda y Empleo del Gobierno de Cantabria, los ayuntamientos de Avilés, Barcelona, Sigüenza (Guadalajara) y Candelaria (Tenerife), así como Vodafone España, El Corte Inglés, Tirme y los grupos Simply, Saica y Promecal, etc.
La ampliación de las redes empresariales y la creación de comunidades que giran en torno a la responsabilidad social de las empresas y que incluyan tanto a las de inserción como a las ordinarias, es fundamental para el mantenimiento del ecosistema empresarial a largo plazo.

La comunicación y difusión de lo que sucede en torno a las empresas de inserción es una pieza importante del ecosistema de la economía social. La difusión del proyecto ERI permite explicar historias de éxito de las que todas las personas, empresas e instituciones pueden aprender. Al mismo tiempo, sirve para atraer a otras personas con talento y empresas para formar parte de la red de colaboración con las empresas de inserción. Por todo ello, el trabajo en red se concibe en FAEDEI como un mecanismo estratégico imprescindible, tanto para la economía social como para las empresas de inserción.

\section{El proceso de acompañamiento en las empresas de inserción}

Uno de los elementos que forma parte indiscutible e identificativo de las empresas de inserción es el trabajo de acompañamiento a la persona que está en el proceso de inserción, a lo largo de todo el periodo que permanece en la empresa. Este trabajo es fundamental para lograr los objetivos de inserción social que se plantean al inicio del proceso y que se plasman en el acuerdo de inserción que se firma con la persona y con los servicios sociales. El papel de acompañamiento nos viene marcado en la Ley 44/2007, para la regulación del régimen de las empresas de inserción, en su artículo 3 que establece el itinerario de inserción socio-laboral y de servicios de intervención y de acompañamiento por medio de empresas de inserción:

1. Las empresas de inserción aplicarán itinerarios de inserción socio-laboral en función de los criterios que establezcan los Servicios Sociales públicos competentes y los Servicios públicos de Empleo, de acuerdo con las propias empresas de inserción, teniendo que ser aceptados dichos itinerarios por la persona en situación de exclusión social contratada, con el objetivo de promover su integración en el mercado laboral ordinario, definiendo las medidas de intervención y acompañamiento que sean necesarias. 


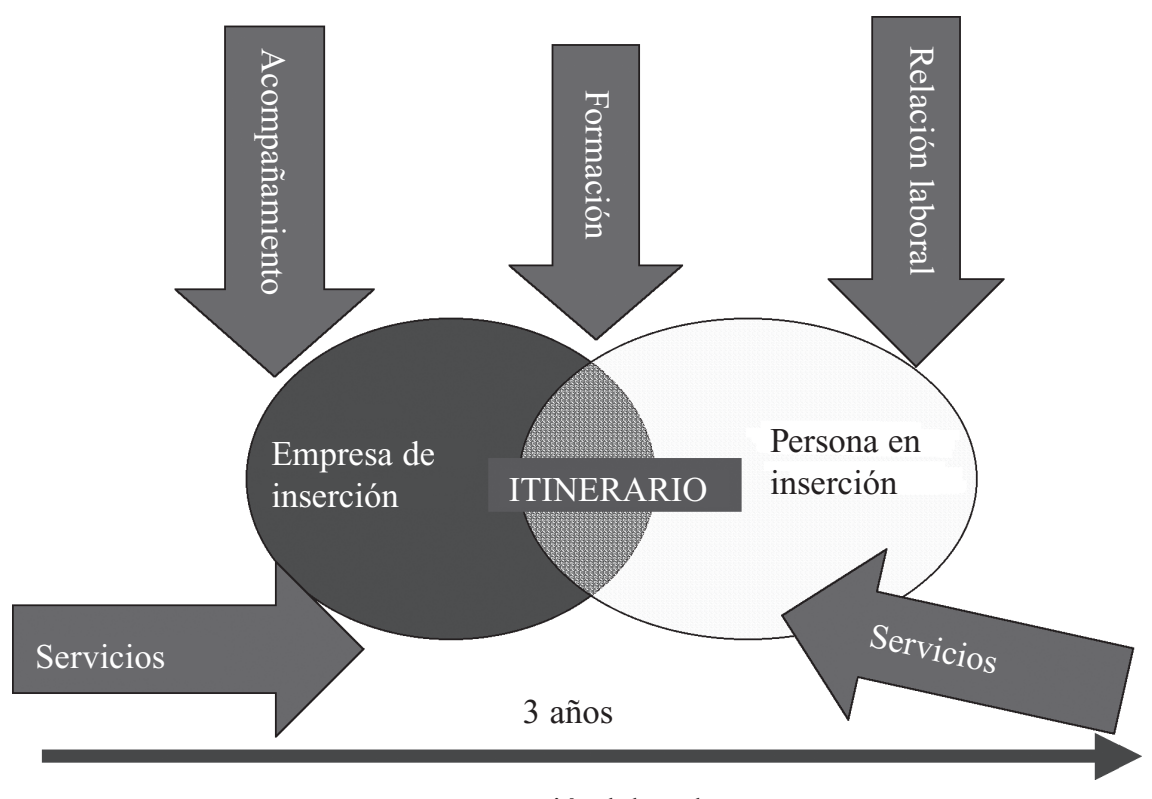

Inserción laboral

Gráfico 1. Elementos del proceso de inserción.

Fuente: Elaboración propia.

2. Las medidas de intervención y acompañamiento consistirán en el conjunto de servicios, prestaciones, acciones de orientación, tutorías y procesos personalizados y asistidos de trabajo remunerado, formación en el puesto de trabajo, habituación laboral y social, encaminados a satisfacer o resolver problemáticas específicas derivadas de la situación de exclusión que dificultan a la persona un normal desarrollo de su itinerario en la empresa de inserción.

En el Gráfico 1 se resumen los elementos que intervienen a lo largo de todo el proceso de la persona en la empresa de inserción, y que va a marcar relaciones, tareas y funciones:

La Ley 44/2007 que regula el régimen de las empresas de inserción también define los papeles que cada actor tiene que cumplir (Cuadro 3).

Según estas funciones, la empresa de inserción tiene que estar en permanente coordinación con los Servicios Sociales públicos, y de forma más temporal con los de empleo. Esta exigencia tiene como consecuencia que la empresa de inserción debe incorporar a su estructura la figura de personal técnico de acompañamiento que va a realizar las funciones de coordinación y dinamización de esta red de apoyo de la persona en inserción.
Esta figura plantea retos para las empresas en diversos planos:

- Nivel organizativo: es necesario encajar sus funciones y tareas enmarcadas dentro del funcionamiento diario productivo de la empresa con las personas que trabajan, con los técnicos de inserción y con la gerencia, así como con los organismos externos como Servicios Sociales públicos y de empleo, entre otros.

- Nivel de recursos humanos: dado el papel que tienen que desempeñar es necesario definir muy bien el perfil del puesto y sobre todo definir las competencias fundamentales mínimas, destacando entre otras la asertividad, un perfil mediador, constructivo y un claro dominio de las competencias sociales. Sin obviar que en cada comunidad autónoma, donde existe apoyo económico parcial para este puesto, viene definida la formación y experiencia mínima requerida para este tipo de profesionales.

- Nivel económico: ya que su retribución no está ligada a un trabajo productivo directo, por lo que se puede considerar un coste añadido para la empresa que hay que contemplar en el presupuesto anual.

- Nivel legal: la empresa de inserción, según el artículo 5 de la Ley, tiene la obligación 


\begin{tabular}{|c|c|c|}
\hline Empresa inserción & Servicios sociales & Servicios de empleo \\
\hline \multirow{2}{*}{$\begin{array}{l}\text { Aplicar los criterios establecidos } \\
\text { por los Servicios Sociales públi- } \\
\text { cos y por los Servicios públicos } \\
\text { de Empleo }\end{array}$} & $\begin{array}{l}\text { Acreditar la situación de exclu- } \\
\text { sión social }\end{array}$ & $\begin{array}{l}\text { Certificar que no ha trabajado } \\
\text { en los } 2 \text { años anteriores }\end{array}$ \\
\hline & $\begin{array}{l}\text { Establecer los criterios para los } \\
\text { itinerarios junto con los Servi- } \\
\text { cios públicos de empleo y la } \\
\text { Empresa de inserción }\end{array}$ & $\begin{array}{l}\text { Establecer los criterios para los } \\
\text { itinerarios junto con los Servi- } \\
\text { cios Sociales públicos y la em- } \\
\text { presa de inserción }\end{array}$ \\
\hline \multirow{3}{*}{$\begin{array}{l}\text { Poner a disposición de la perso- } \\
\text { na en inserción medidas de in- } \\
\text { tervención y acompañamiento }\end{array}$} & Seguimiento de los itinerarios & \multirow[t]{2}{*}{ Seguimiento de los itinerarios } \\
\hline & $\begin{array}{l}\text { Determinar si las faltas de asis- } \\
\text { tencia o puntualidad son justifi- } \\
\text { cadas }\end{array}$ & \\
\hline & $\begin{array}{l}\text { Informar sobre la adecuación de } \\
\text { las prórrogas }\end{array}$ & $\begin{array}{l}\text { Certificar la formación adquiri- } \\
\text { da y, en su caso, la correspon- } \\
\text { dencia entre la experiencia ad- } \\
\text { quirida y las competencias } \\
\text { descritas en los certificados de } \\
\text { profesionalidad del Sistema Na- } \\
\text { cional de Cualificaciones }\end{array}$ \\
\hline
\end{tabular}

Cuadro 3. Funciones de los actores implicados

Fuente: Elaboración propia.

de presentar anualmente un balance social donde aparezcan las acciones de acompañamiento e inserción que ha realizado con cada persona durante ese periodo, con el riesgo de perder la calificación sino lo hace.

El personal de acompañamiento técnico es una figura clave para lograr los objetivos de inserción social y laboral de las personas, que es el fin último de las empresas de inserción. Su trabajo se realiza a lo largo de todo el itinerario de inserción, por eso, desde la Federación Estatal (FAEDEI), como parte del trabajo en red que viene desarrollando, y con la Asociación Española de Recuperadores de la Economía Social y Solidaria (AERESS) hace tres años se puso en marcha un grupo de trabajo formado por personal técnico de empresas de inserción de todo el Estado, con la ayuda inestimable del Departamento de Orientación y Didáctica de la Facultad de Filosofía y Ciencias de la Educación de la Universidad de Valencia. Durante estos años hemos ido recabando información, analizando, evaluando y consensuando las diferentes fases que una persona en inserción va pasando a lo largo de su estancia en la empresa.
Uno de los objetivos de este grupo es el de desarrollar un «modelo de itinerario de acompañamiento a la inserción», común para todas las empresas de inserción que ya existen o las que se vayan a constituir. Queremos que sea una herramienta ágil, útil, flexible, dinámica y que pueda servir tanto al personal técnico de acompañamiento como a los de producción, como actores fundamentales en el día a día de la empresa.

Para lograrlo hemos desarrollado una metodología de trabajo mixta, presencial y a distancia, que contempla un plan de trabajo de tres años con un programa operativo anual. Durante el primer año hemos recabado información de las empresas a través de cuestionarios, estudio de caso, herramientas y metodología que las empresas están desarrollando. El grupo analizó los resultados de 30 empresas, sistematizó y tuvo lugar el primer seminario de trabajo con la asistencia de 60 empresas y 110 participantes, en el que trabajamos los resultados detectando las principales áreas de mejora metodológica. Las principales conclusiones fueron:

- Consensuar las tres grandes etapas del proceso: acogida y evaluación inicial; segui- 
miento y transición al mercado laboral normalizado.

- Constatación del amplio trabajo llevado a cabo en las empresas de inserción durante la fase de acogida, obteniendo importantes instrumentos metodológicos, sistematización de la información etc. Sin embargo, en la última fase destinada a la transición en el mercado laboral ordinario, existen menos instrumentos comunes siendo también la metodología empleada más variada.

- Igualmente, se identificó como campo de trabajo importante, la fase de seguimiento, con el objetivo de unificar instrumentos y definir la mejor metodología, que posibilite la transición con eficacia.

- Finalmente se consideró la necesidad de definir, seleccionar e implementar un sistema de evaluación para la persona en inserción que recoja su experiencia en la empresa.

Tras este seminario, el grupo desarrolló el año siguiente los elementos detectados y se preparó el II Seminario centrado en la fase de tránsito al mercado laboral normalizado. Para ello, se preparó un cuestionario que se envió a todas las empresas de inserción y de su devolución extrajimos los elementos fundamentales para profundizar en el segundo encuentro, introduciendo una metodología más participativa para el debate y el consenso de las conclusiones. El encuentro se desarrolló en Avilés y contó con la participación de 125 personas. El trabajo se organizó en tres áreas: indicadores, acciones y herramientas utilizadas en el trabajo diario durante esta fase. Partiendo de casos reales, instrumentos, indicadores y acciones que previamente habían enviado las empresas al grupo, se trabajaron en detalle cada uno de los puntos. Posteriormente se hizo un trabajo de sistematización y elaboración de conclusiones de cada apartado aprobado en asamblea, consensuándose las acciones, indicadores y herramientas de trabajo para el que será el Manual de acompañamiento de las empresas de inserción.

El trabajo continúa este año, centrándose en la fase de seguimiento, con la misma metodología empleada hasta el momento, partir de la experiencia de las empresas de inserción, independientemente de la actividad económica desempeñada, para poder consensuar la mejor metodología basada en la experiencia. La Federación ha redactado la Introducción al manual que recogerá los valores, fundamentos y razón de ser de las empresas de inserción.

Este trabajo de acompañamiento individual, durante el tiempo que la persona realiza su actividad en la empresa de inserción, es el eje sobre el que articulamos el resto de actividades, por ello es fundamental el papel que el técnico o la técnica va realizando durante su itinerario y es el que nos sirve para ir valorando la mejora no sólo en la empleabilidad de la persona, sino del conjunto de elementos tanto personales, como familiares y del entorno más cercano, que están incluidos en su itinerario personal y que contribuyen al éxito de su inserción social y laboral una vez que abandone la empresa.

Para ello es fundamental realizar un trabajo de coordinación con los otros agentes sociales activos en el proceso: Servicios Sociales y de empleo, empresas ordinarias, sistemas educativos, sanitarios, etc. Este trabajo conjunto, es una de las características que posibilitan que las Empresas de Inserción sean una herramienta eficaz y eficiente para la inserción social y laboral de las personas con dificultades de integración, además de su contribución a la economía del estado.

\section{Conclusiones}

Las empresas de inserción son un modelo de economía social solidaria de eficacia reconocida para la inserción sociolaboral de personas en situación o riesgo de exclusión social. A nivel estatal estas empresas representadas por la Federación de Asociaciones Empresariales de Empresas de Inserción (FAEDEI) son un instrumento importante para la puesta en práctica de las políticas activas de empleo. Uno de los retos más importantes que tenemos actualmente en FAEDEI es dar a conocer cómo las empresas de inserción complementan los programas y políticas públicas de las distintas administraciones y suponen un ahorro en gasto social.

La actual crisis económica y la reformulación del estado de bienestar supone que cada día crezca y se consoliden más colectivos en situación o riesgo de exclusión, entre otros, el número de personas perceptoras de rentas mínimas de inserción o de cualquier otra prestación de igual o similar naturaleza y personas 
que no puedan acceder a las prestaciones por falta del período exigido de residencia o empadronamiento o haber agotado el período máximo de percepción de prestaciones legalmente establecido. Por ello, es necesario poner de manifiesto «la falta de madrinas y padrinos» reconocidos para muchos de los colectivos en situación o riesgo de exclusión, frente a la gran concienciación social con las personas con discapacidad, otros colectivos como los menores procedentes de instituciones de menores, las personas con problemas de drogodependencia o trastornos aditivos y las personas internas en centros penitenciarios, no cuentan con el mismo respaldo social.

Las empresas de inserción realizan un trabajo técnico, metodológico, de acompañamiento y de trabajo en coordinación con los otros agentes sociales, que permite mejorar la empleabilidad de las personas, y las circuns- tancias personales y familiares, que favorezcan una inserción social y laboral posterior.

Resulta fundamental dar a conocer los productos y servicios de las empresas de inserción a todos los potenciales colaboradores y/o compradores: con las administraciones públicas y las empresas públicas, a las que se les pide la reserva de mercado y /o la introducción de cláusulas sociales en la adjudicación de obras y servicios. Con las empresas privadas queremos establecer acuerdos de colaboración directos que permitan crear sinergias.

Para ello, la creación de redes y comunidades que incluyan a las empresas de inserción y las empresas ordinarias es fundamental para el mantenimiento del ecosistema empresarial a largo plazo. El trabajo en red que realizamos se convierte en un mecanismo estratégico imprescindible tanto para la economía social como para las empresas de inserción.

\section{Referencias bibliográficas}

Comisión Europea. (2011). Comunicación de la Comisión al Parlamento Europeo, al Consejo, al Comité Económico y Social Europeo y al Comité de las Regiones. Nueva Estrategia de la UE 2011- 2014 sobre la responsabilidad social de las empresas (COM(2011) 681 final, de 25/10/2011). Bruselas: Comisión Europea. Recuperado de

http://eur-lex.europa.eu/LexUriServ/LexUriServ.do?uri=COM:2011:0681:FIN:ES:PDF

Comisión Europea. (2010). Comunicación de la Comisión: Europa 2020 .Una estrategia para un crecimiento inteligente, sostenible e integrador (COM (2010) 2020 de 3/3/2010). Bruselas: Comisión Europea. Recuperado de http://ec.europa.eu/commission_2010-2014/president/ news/documents/pdf/20100303_1_es.pdf.

Comisión Europea. (2010). Comunicación sobre la Unión por la innovación, (COM (2010) 546 final de 6/10/ 2010). Bruselas: Comisión Europea. Recuperado de http://eur-lex.europa.eu/LexUriServ/LexUriServ.do?uri=COM:2010:0546:FIN:es:PDF

Comisión Europea. (2010). Comunicación La Plataforma Europea contra la Pobreza y la Exclusión Social: Un marco europeo para la cohesión social y territorial (COM (2010) 758 final de 16/09/2010). Bruselas: Comisión Europea. Recuperado de: http://eur-lex.europa.eu/LexUriServ/LexUriServ.do?uri=OJ:C:2011:166:0018:0022:ES:PDF

Comisión Europea. (2011). Acta del Mercado Único. Doce prioridades para estimular el crecimiento y reforzar la confianza (COM (2011) 206 final de 13/4/ 2011). Bruselas: Comisión Europea. Recuperado de: http://eur-lex.europa.eu/LexUriServ/LexUriServ.do?uri=COM:2011: 0206:FIN:es:PDF

Ley 5/1982, de 7 de abril, de Integración Social de Minusválidos. Boletín Oficial del Estado, 103, de 30/4/1982, pp. $11106-11112$.

Ley 5/2011, de 29 de marzo, de Economía Social. Boletín Oficial del Estado, 76, de 30/3/ 2011, pp. 33023-33033.

Ley 44/2007, de 13 de diciembre, para la regulación del régimen de las empresas de inserción. Boletín Oficial del Estado, 299 de 14/12/2007, pp. 51331 a 51339.

Real Decreto-Ley 4/2013, de 22 de febrero, de medidas de apoyo al emprendedor y de estímulo del crecimiento y de la creación de empleo. Boletín Oficial del Estado, 47, pp. 15219-15271. 